\title{
NANOSCALE TOPOGRAPHY CONTROL FOR THE FABRICATION OF ADVANCED DIFFRACTIVE OPTICS
}

\author{
J. Alexander Liddle, ${ }^{*}$ Farhad Salmassi, Patrick P. Naulleau and Eric M. Gullikson \\ Center for X-Ray Optics, Lawrence Berkeley National Laboratory, 1 Cyclotron Road, Berkeley, \\ CA 94720, USA
}

keywords: Nanofabrication, electron-beam, lithography, EUV, diffractive optics

\begin{abstract}
Grey-scale electron beam lithography has been used to create high-efficiency $(63 \%$, output normalized) blazed gratings suitable for use at extreme ultraviolet (EUV) wavelengths (i.e. $13.4 \mathrm{~nm}$ ). The total blaze height at these wavelengths is $\approx 7 \mathrm{~nm}$. The surface topography was generated in a single processing step in hydrogen silsesquioxane (HSQ). This material converts to $\mathrm{SiO}_{2}$ upon exposure, forming a robust substrate for subsequent operations, unlike conventional organic resists. The HSQ is overcoated with a $\mathrm{Mo} / \mathrm{Si}$ multilayer to provide reflectivity at EUV wavelengths. The grating efficiency is determined by the fidelity of the profile to the ideal and by the surface roughness of the HSQ. A region of the resist response curve was identified that enabled sufficient topography to be generated while maintaining the surface roughness of the resist below $2.5 \mathrm{~nm}$ RMS. Large area $\left(0.5 \times 2.0 \mathrm{~mm}^{2}\right)$ gratings were fabricated, and the delivered dose profile was adjusted during the course of the exposure to compensate for observed delay-time/reciprocity effects in HSQ.
\end{abstract}

\section{INTRODUCTION}

High efficiency gratings in the soft X-ray and extreme ultraviolet (EUV) regimes are desirable in many applications as monochromators. In this paper we report on the fabrication of a highly efficient blazed grating for use as a spectral purity filter in the lithographically interesting wavelength range centered around $13.4 \mathrm{~nm}$. The grating is coated with a Mo/Si reflective multilayer and used at near-normal incidence in first order.

Currently available sources for EUV lithography systems, whether synchrotron ${ }^{2}$ or laser ${ }^{3} /$ pinch $^{4} /$ discharge ${ }^{5}$ generated plasma based are broadband. Because there are no materials that are transparent at EUV wavelengths, only reflective optical systems can be employed. This means that essentially all wavelengths, down to the deep-UV, from the source can propagate through the system to the wafer. However, in order to minimize the heat load on the optics infrared (IR) radiation must be prevented from passing through, while ultraviolet (UV) wavelengths cannot be permitted to travel to the wafer plane and expose the resist. For these reasons a spectral purity filter is required to eliminate all but the desired EUV wavelengths, and it must transmit these with very high efficiency.

A blazed grating (Figure 1) can perform this function effectively. When the angle of a diffracted order coincides with the specular reflection angle from a single blaze, then essentially all of the power is contained in that order. ${ }^{6}$ The conditions are:

\footnotetext{
*e-mail: jaliddle@1bl.gov
} 


$$
\begin{aligned}
& a\left(\sin \theta_{m}-\sin \theta_{i}\right)=m \lambda, \quad \theta_{i}-\theta_{r}=2 \gamma \\
& \text { For } 1 \text { st order, } m=1 \\
& \gamma=h / a, \quad \theta_{m}=\theta_{r} \Rightarrow h \cong \lambda / 2 \cos \theta_{i}
\end{aligned}
$$

For approximately normal incidence the blaze height, $h$, is therefore half the wavelength of the diffracted light, or $6.7 \mathrm{~nm}$ for the desired $13.4 \mathrm{~nm}$ EUV radiation. We note that for the longer IR and UV wavelengths the effect of the topography is negligible and that this light is specularly reflected from the grating plane.

\section{GRATING DESIGN}

The overall goal of the grating design and fabrication process is to produce a filter that is more than $50 \%$ efficient - better than that achieved by a simple transmission-grating filter. ${ }^{7}$

In order to maintain approximately normal incidence and to make fabrication easier, the grating is designed with a $1 \mu \mathrm{m}$ pitch, producing a $1^{\text {st }}$ diffracted order at $13.4 \mathrm{mrad}$. This relatively small angular separation is sufficient to permit the construction of an entrance slit that blocks all of the specular reflection. The Mo/Si multilayer coating has a small bandpass (typical values are in the range of $0.6 \mathrm{~nm}$ FWHM at $13.4 \mathrm{~nm}$ ) and effectively filters out those wavelengths that are not dispersed outside the entrance slit by the grating.

We note that, for the multilayer to be reflective at the angle of incidence, $\theta_{\mathrm{i}}$, the period, $d$, is given approximately by

$$
d=\lambda /\left(2 \sin \left(90-\theta_{i}\right)\right)=\lambda /\left(2 \cos \theta_{i}\right)=h .
$$

This means that the period of the multilayer coating is matched to the height of the blaze. It is also apparent that, since $\theta_{i}=\cos ^{-1}(\lambda / 2 h)$, small changes in blaze height will necessitate large changes to the angle of incidence to maintain overall efficiency at a given wavelength.

The efficiency of a binary diffractive optical structure improves as the number of steps is increased, making it more closely approximate the ideal kinoform. ${ }^{8}$ In this instance we have chosen 8 steps which, in the absence of other losses, will yield a structure that is $95 \%$ efficient. This implies that each step will differ by less than $1 \mathrm{~nm}$ from the adjacent ones.

In addition to controlling the blaze height and individual step heights at the nanometer scale, the surface roughness of the structure is also critical. The absolute reflectivity loss of the multilayer coating is approximately $2 \%$ per $0.1 \mathrm{~nm}$ RMS substrate roughness. ${ }^{9}$ It is therefore desirable to keep the roughness of the structure below $1 \mathrm{~nm}$.

\section{GRATING FABRICATION}

Nanometer-scale topographies are difficult to manufacture using ruling engines so alternative methods such as grey-scale e-beam lithography have been explored. Although this technique has been used in the visible light regime, ${ }^{10,11,12}$ there has been only one attempt at these shorter wavelengths. ${ }^{13}$

The strict demands on the surface profile, together with the desire to minimize the number of processing steps led us to use hydrogen silsesquioxane (HSQ), which converts to $\mathrm{SiO}_{2}$ upon exposure. $^{14}$ HSQ has appropriate contrast and roughness characteristics and, once it is exposed, is robust enough to withstand multilayer deposition. This obviates the need for the development of an etch transfer process to convert the resist profile into that of an underlying substrate material. 
In order to determine the appropriate set of doses to generate the grating profile, we measured, in detail, a contrast curve for an $80 \mathrm{~nm}$ thick HSQ film. The film was prepared by spinning a $3.6 \%$ solution of $\mathrm{HSQ}^{15}$ dissolved in methyl isobutyl ketone at $1000 \mathrm{rpm}$ on a 4 " wafer. The spin casting solvent was removed by oven baking at $170 \mathrm{C}$ for 5 minutes. The film was exposed in areas of $1000 \times 200 \mathrm{~nm}$ in a geometric progression of 150 doses from 250 $\mu \mathrm{C} / \mathrm{cm}^{2}$ to $1090 \mu \mathrm{C} / \mathrm{cm}^{2}$, and the film thickness was measured by AFM. ${ }^{16}$ The film roughness was also measured at intervals. The results are shown in Figure 2.

It is clear that the required topography can be generated by an infinite set of doses: based on the variation of roughness with dose, however, it is clear that we must operate at the highest doses possible to achieve the desired low surface roughness.

In order to determine the actual doses to be used, we first fit the contrast curve using a function of the form

$$
t(\text { Dose })=t_{0}\left(1-\frac{A}{\text { Dose }}-\frac{B}{D_{o s e}^{2}}\right)
$$

Where $t$ (Dose) is the film thickness remaining at a given dose, $t_{0}$ is the initial film thickness and $A$ and $B$ are fitting parameters. We note that this fit is not based on a physical model, but provides a convenient functional form. Once the fit has been determined, the desired topography can be converted into a dose profile, $\operatorname{Dose}(x)$. The final step is to adjust the incident dose to account for proximity effects. This is a straightforward procedure in this case because the grating period, $1 \mu \mathrm{m}$, is short in relation to the backscatter range at $100 \mathrm{keV}$ of $30 \mu \mathrm{m}$, and because the grating covers an area, $500 \mu \mathrm{m} \times 2000 \mu \mathrm{m}$, that is much larger than the backscatter range. Under these conditions the correction is simply a dose offset, $\Delta$, determined by solving the following:

$$
\operatorname{Dose}(x)=(\operatorname{Dose}(x)-\Delta)+\frac{\eta}{a} \int_{0}^{a}(\operatorname{Dose}(x)-\Delta) d x \text {. }
$$

Where $a$ is the grating period and $\eta$ is the effective backscatter coefficient.

Figure 3 shows a comparison of calculated and measured blaze heights as a function of the fraction of the nominal incident dose. Scaling the incident doses by a multiplier is an effective way of fine tuning the blaze height.

Two features are apparent from Figure 3: first, there is a consistent offset between the blaze heights measured before and after coating, and second, there is a substantial offset between the calculated and measured blaze heights.

The increase in blaze height after coating is an artefact of the AFM measurement, as demonstrated in Figure 4. The surface roughness and measured topography are strongly dependent on the size of the AFM tip, with the conventional pyramidal Si tip failing to capture accurately the fine scale roughness and topography of the exposed and developed HSQ, particularly as the surface roughness increases towards the lower doses of a grating period. Both conventional and nanotube tips accurately reflect the topography of the coated gratings because of the very low surface roughness of the multilayer.

More serious is the discrepancy between the calculated and measured blaze heights. The contrast curves are determined by measuring small exposed areas, while the grating comprises $100 \mu \mathrm{m} \times 100 \mu \mathrm{m}$ squares. There is a significant difference in exposure time for the two, so we 
investigated the possibility that the time delay between introducing the coated wafer into vacuum and the exposure might be responsible. Although there are slight differences in the resist in terms of the total resist height, the topography produced appears to independent of delay time. In an attempt to clarify the situation, we measured contrast curves in which the measured features had received a baseline additional dose, either as large area exposure, or as an exposure over the same area as the measured features.

The contrast curves shown in Figure 5 clearly shift in the direction of greater or lesser sensitivity depending on exactly how the dose is delivered, indicating that there is a failure of reciprocity in this material. This effect is currently under investigation, but the sensitivity of the resist appears dependent on the extent of any adjacent exposed material. The implications for this in terms of writing large areas are illustrated in Figure 6 a). An empirical correction has been applied to the grating shown in Figure 6 b), resulting in a much more uniform topography.

\section{GRATING CHARACTERIZATION}

The coated gratings were evaluated using the calibration and standards bend-magnet beamline 6.3.2 at the Lawrence Berkeley National Laboratory Advanced Light Source which has a $\lambda / \Delta \lambda$ resolution of 1400 . Figure $7 \mathrm{~b}$ ) shows the results of a diffraction pattern measurement at the multilayer's most efficient wavelength. ${ }^{17}$ The relative diffraction efficiency of $63 \%$ is the highest reported so far. We note that this is still well below that predicted theoretically, for a perfect blazed grating. As can be seen from the diffraction data, there is a considerable amount of power $(20 \%)$ in orders other than the -1 , due to the difference between the actual and ideal profiles. The asymmetry in the profile results from the small, but finite, slope on the back of the blaze. The remaining power is not diffracted but scattered, as a result of the roughness of the grating surface.

\section{CONCLUSIONS}

We have fabricated a highly efficient blazed grating for use at EUV wavelengths, identifying the conditions necessary for nanometer-scale topography control in HSQ, including corrections for time/dose dependent effects. The efficiency could be further enhanced by making the surface of the HSQ smoother, before multilayer deposition, possibly by means of an annealing step, by increasing the number of dose steps, or by attempting to find development conditions that yield a less rough surface. The techniques developed here are generally useful for any type of structure where very fine scale topography is required and will find particular application to diffractive optics in the EUV.

\section{ACKNOWLEDGMENTS}

This work was supported in part by DARPA under contract number MDA972-98-C-0007 and by the Director, Office of Science, Office of Basic Energy Sciences, Materials Science and Engineering Division, U.S. Department of Energy under Contract Nos. DE-AC03-76SF00098 and W-31-109-ENG-38.

\section{REFERENCES}

${ }^{1}$ J.A.R. Samson and D.L. Ederer in "Vacuum Ultraviolet Spectroscopy", p27, p347, Academic Press, San Diego (2000) 
${ }^{2}$ D. Attwood, "Soft X-rays and Exteme Ultraviolet Radiation, Principles and Applications", pp123-188, Cambridge University Press, Cambridge (2000)

${ }^{3}$ W.P. Ballard, L.J. Bernardez, R.E. Lafon, R.J. Anderson, Y. Perras, A. Leung, H. Shields, M.B. Petach, R.J. StPierre, R. Bristol, Proc. SPIE, 4688302 (2002)

${ }^{4}$ M.W. McGeoch, Proc. SPIE, 3997861 (2000)

${ }^{5}$ N.R. Fornaciari, H. Bender, D. Buchenauer, J.L. Dimkoff, M.P. Kanouff, S. Karim, C. Romeo, G.M. Shimkaveg, W.T. Silfvast and K.D. Stewart, Proc. SPIE, 4688110 (2002)

${ }^{6}$ S.G. Lipson, H. Lipson and D.S. Tannhauser, "Optical Physics" $3^{\text {rd }}$ ed., pp230-232, Cambridge University Press, Cambridge (1995)

${ }^{7}$ P.P. Naulleau, W.C. Sweatt, and D.A. Tichenor, Optics Communications, 21431 (2002).

${ }^{8}$ H.P Herzig, ed., "Micro-Optics, Elements, Systems and Applications", p5, Taylor and Francis, London (1997)

${ }^{9}$ P.B. Mirkarimi, S. Bajit and M.A. Wall, Appl. Opt., 391617 (2000).

${ }^{10}$ M.T. Gale in [8], pp87-126

${ }^{11}$ X-C. Yuan, W.X. Yu, W.C. Cheong and N.Q. Ngo, J. Phys. D: Appl. Phys., 35 L81 (2002)

${ }^{12}$ M. Kalus, M. Frey, L.M. Buchmann, K. Reimer and B. Wagner, Microelec. Eng. 41/42 461 (1998)

${ }^{13}$ P.P. Naulleau, E.H. Anderson, E.M. Gullikson and J. Bokor, Optics Communications, 20027 (2001).

${ }^{14}$ F. van Delft, J. Weterings, A. van Langen-Suurling, H. Romijn, J. Vac. Sci. Technol., B18 3419 (2000)

${ }^{15}$ Dow Corning, FOX 15

${ }^{16}$ Digitial Instruments Inc. DI3100 Nanoscope

${ }^{17}$ P.P. Naulleau 


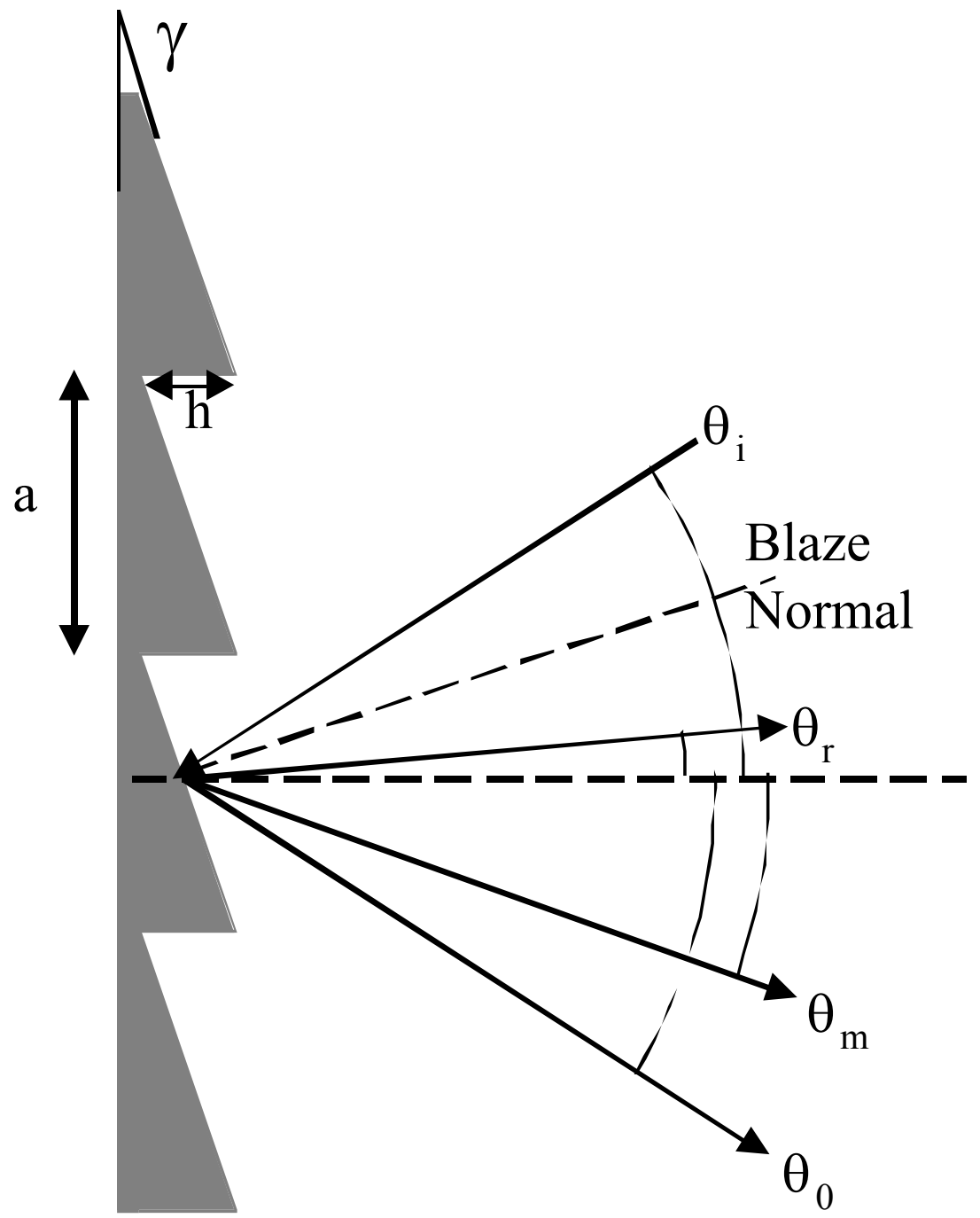

Figure 1. Illustration of a blazed grating, showing the incident light, $\theta_{i}$, the zero-order, specularly reflected light, $\theta_{0}$, the $\mathbf{m}^{\text {th }}$ order diffracted light, $\theta_{m}$, and the direction of specular reflection from an individual blaze surface, $\theta_{r}$. Maximum efficiency in a diffracted order is achieved when $\theta_{m}$ and $\theta_{\mathbf{r}}$ are coincident. $\gamma=\mathbf{h} / \mathbf{a}$. 
a)

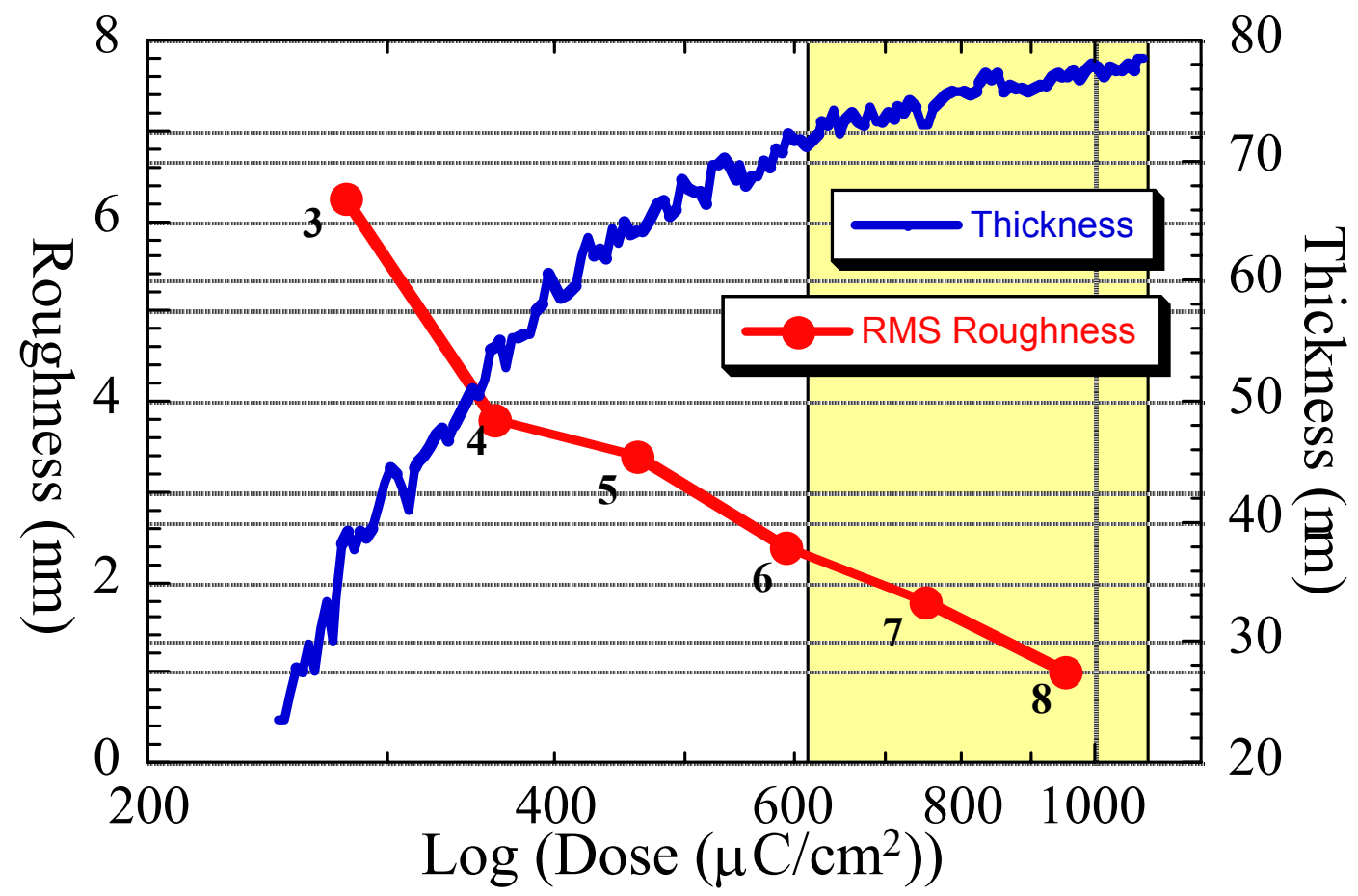

b)
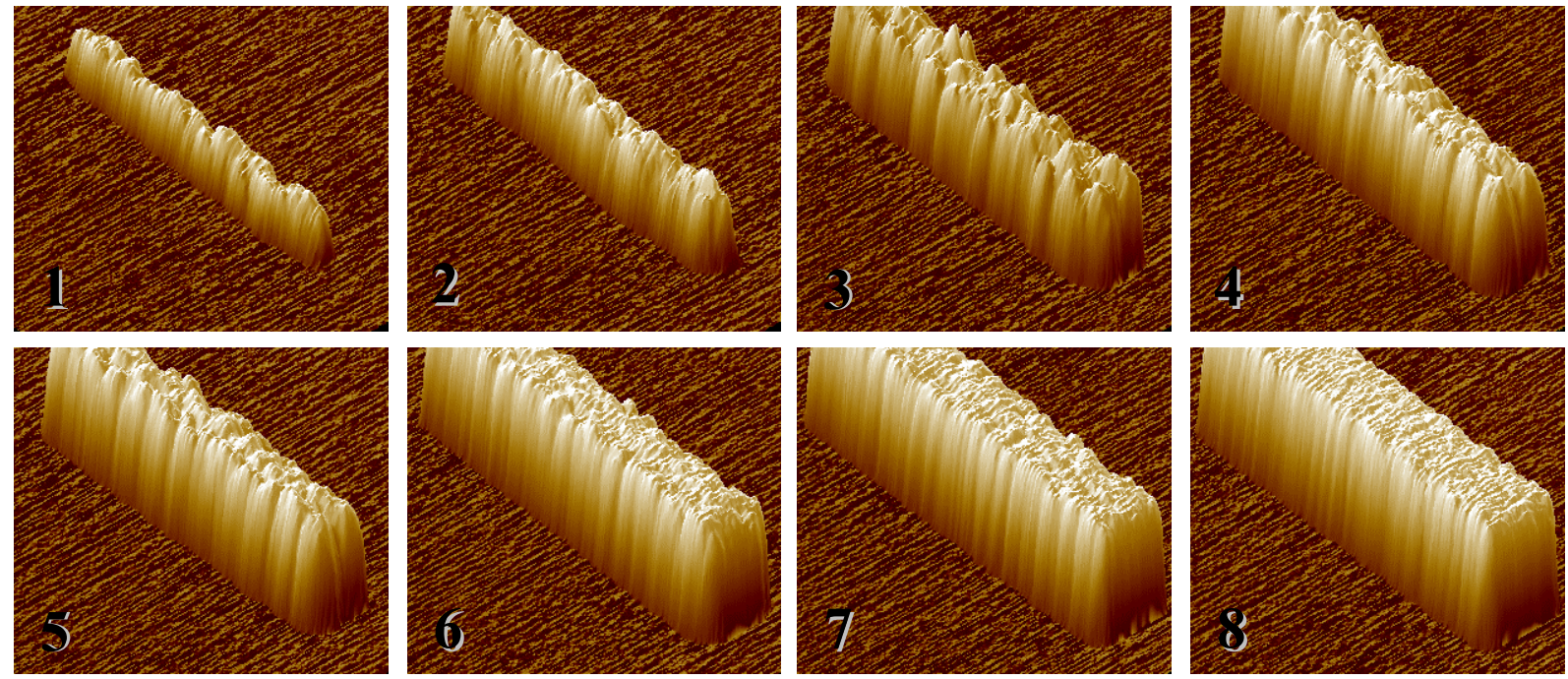

Figure 2. a) Contrast (thickness vs dose) curve for a sample of HSQ determined using a series of 125 doses. The thickness repeatability is $\pm 2 \mathbf{n m}$ The potential operating regime for grating fabrication is shaded. The surface roughness is also plotted for a selection of doses $(1-8)$, with the corresponding images shown in b). The repeatability of the roughness measurements is $\pm 0.3 \mathrm{~nm}$. Both the resist thickness and roughness are measured by AFM using a conventional pyramidal Si tip. 


\section{Height (nm)}

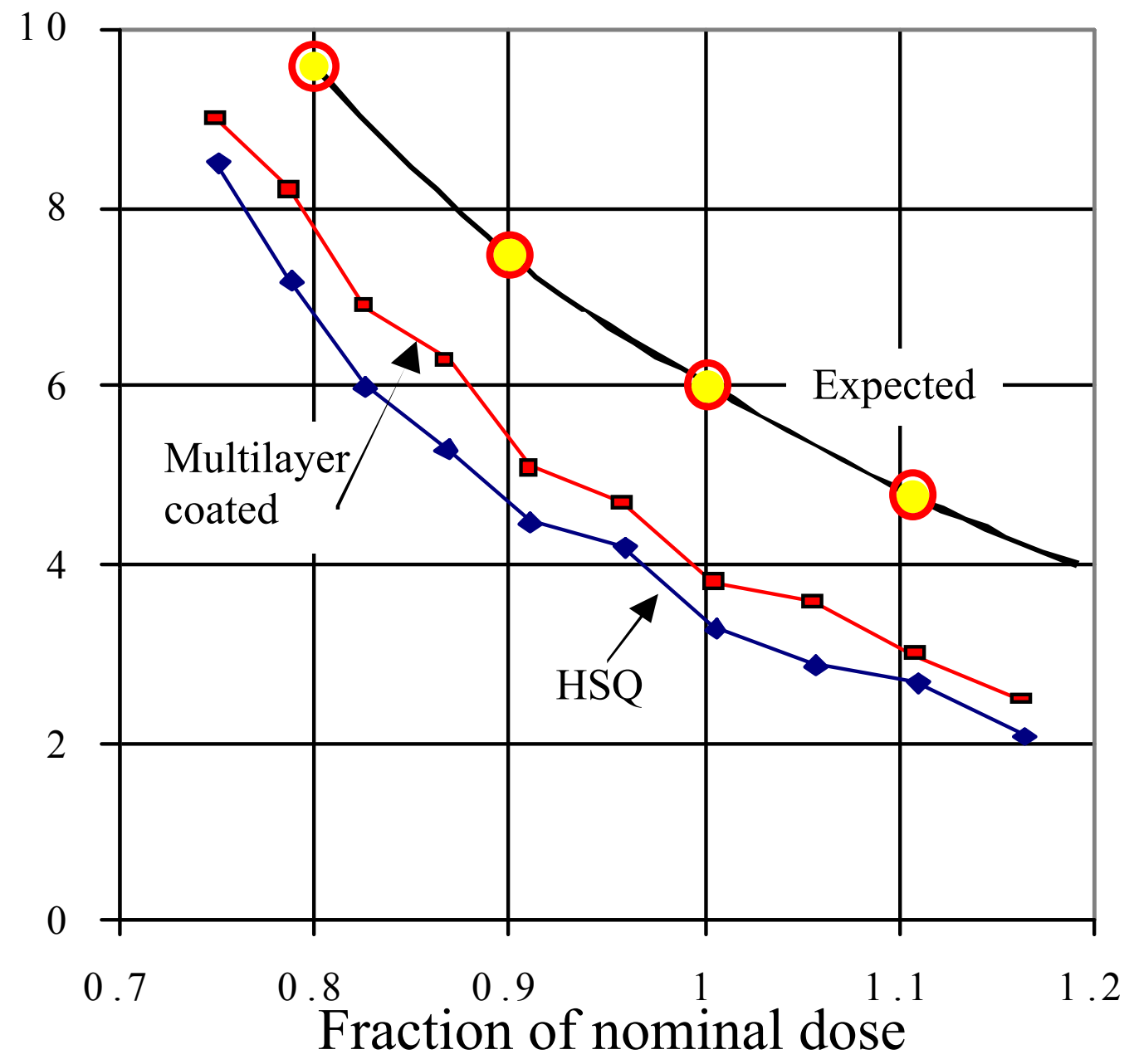

Figure 3. Calculated versus measured topographies for a blazed grating as a function of the fraction of the nominal incident dose. Note that the measured topographies are significantly less than the calculated ones, and that there is a consistent offset between the blaze heights measured before and after multilayer coating. 
a)

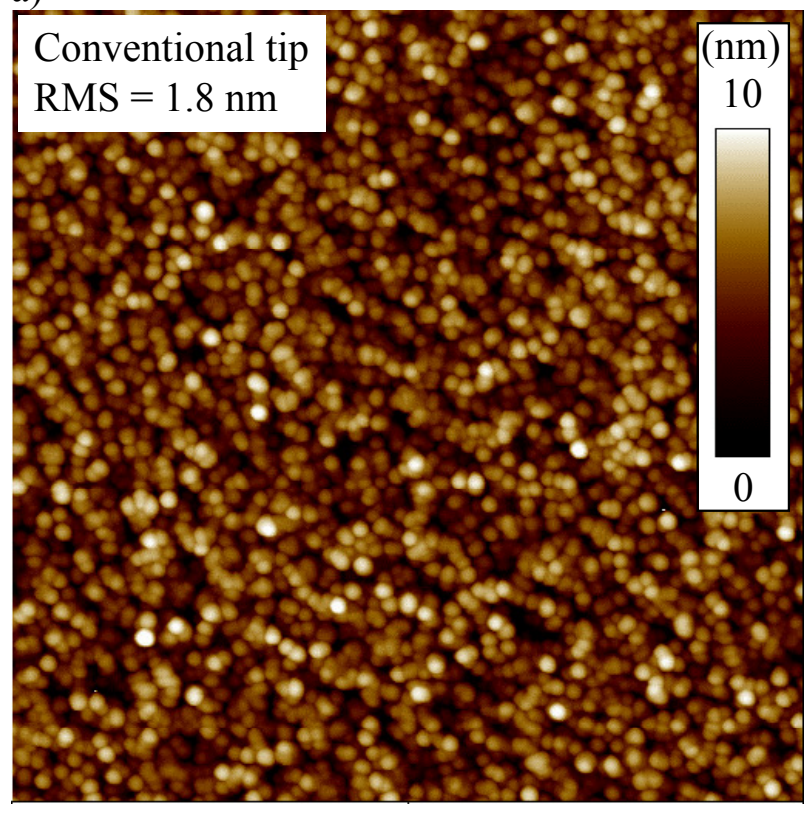

b)

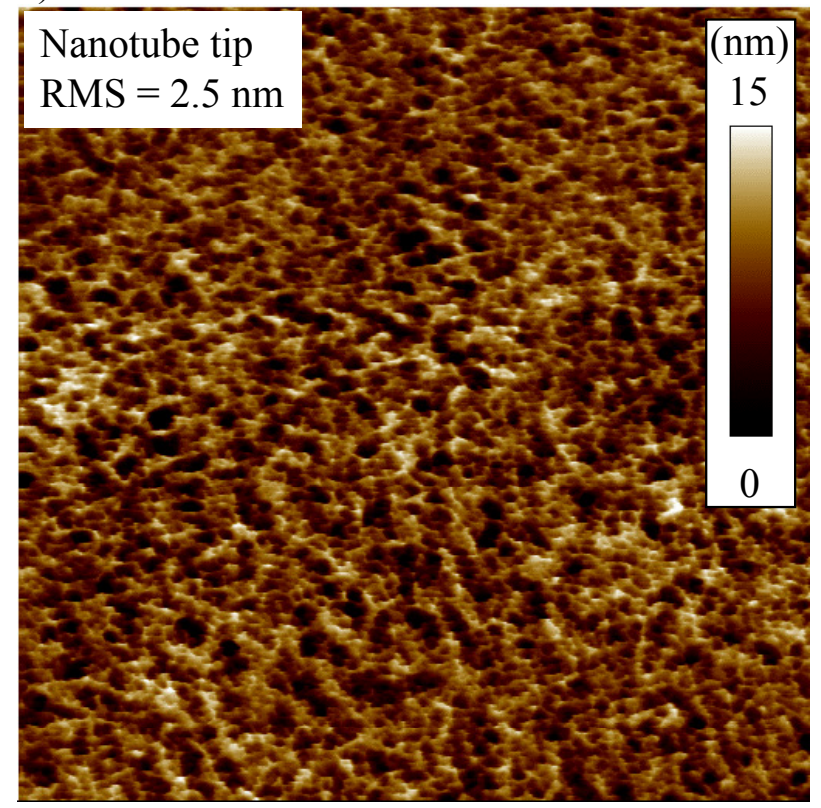

Figure 4. a) AFM scan of an exposed and developed region of HSQ with a conventional pyramidal Si tip. b) AFM scan of a similar area using a carbon nanotube tip. Note the substantial increase in the measured surface roughness from $1.8 \mathrm{~nm}$ RMS to $2.5 \mathrm{~nm}$ RMS. 


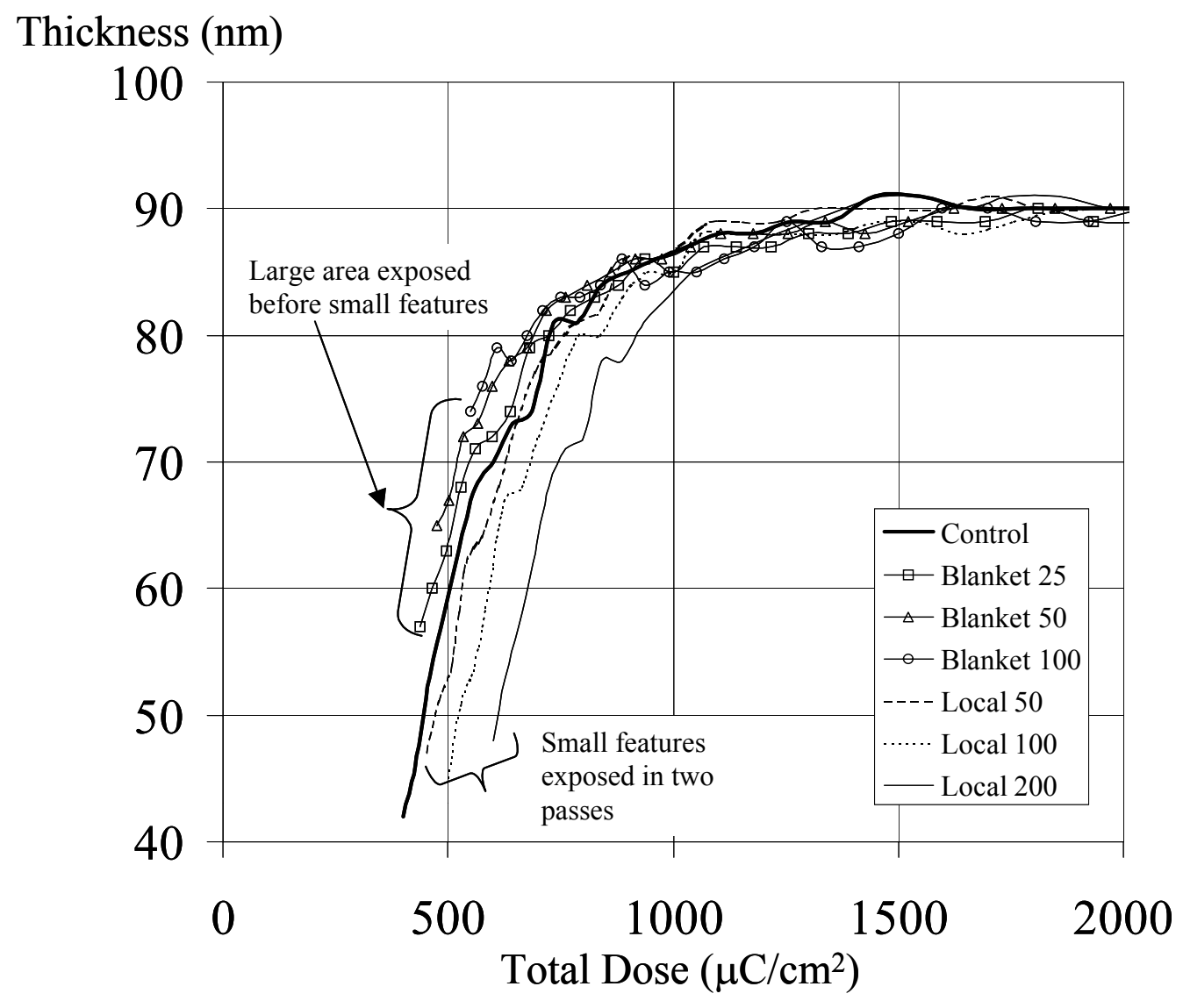

Figure 5. Contrast curves determined by AFM measurements of the heights of a series of twenty-five $1 \mu \mathrm{m}$ features. The features measured in the curves to the left of the control received part of their dose locally in $1 \mu \mathrm{m}$ squares at levels of 25,50 and $100 \mu \mathrm{C} / \mathrm{cm}^{2}$ respectively, while those to the right received part of the dose as a result of a large area backround exposure at levels of 50,100 and $200 \mu \mathrm{C} / \mathrm{cm}^{2}$. The resist responds as if more sensitive when it receives a large background dose, but as if less sensitive when the additional dose is local to the features to be measured. 

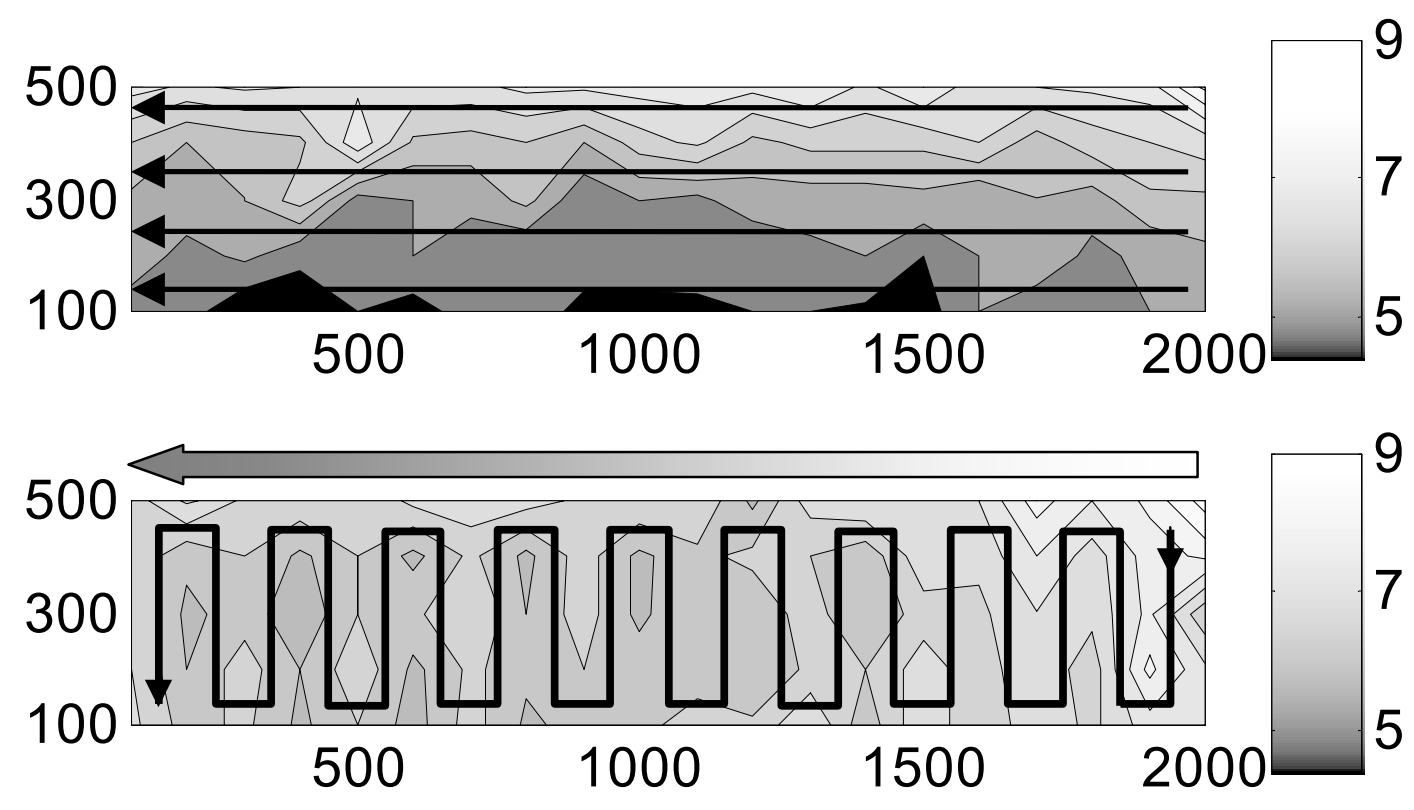

Figure 6. AFM measurements of blaze height taken at the center of each $100 \mu \mathrm{m} \times 100 \mu \mathrm{m}$ cell in a $500 \mu \mathrm{m} \times 2000 \mu \mathrm{m}$ grating written as a sequence of $100 \mu \mathrm{m} \times 100 \mu \mathrm{m}$ cells in the order shown. Note that there is a gradual decrease in the measured topography as the exposure proceeds. b) A similar grating written in the order shown, with a decrease in nominal dose of 0.9983 per $100 \mu \mathrm{m} \times 100 \mu \mathrm{m}$ cell - the final cell receives 0.83 the dose of the initial cell. Note that the topography is much more uniform over the grating area. 
a)

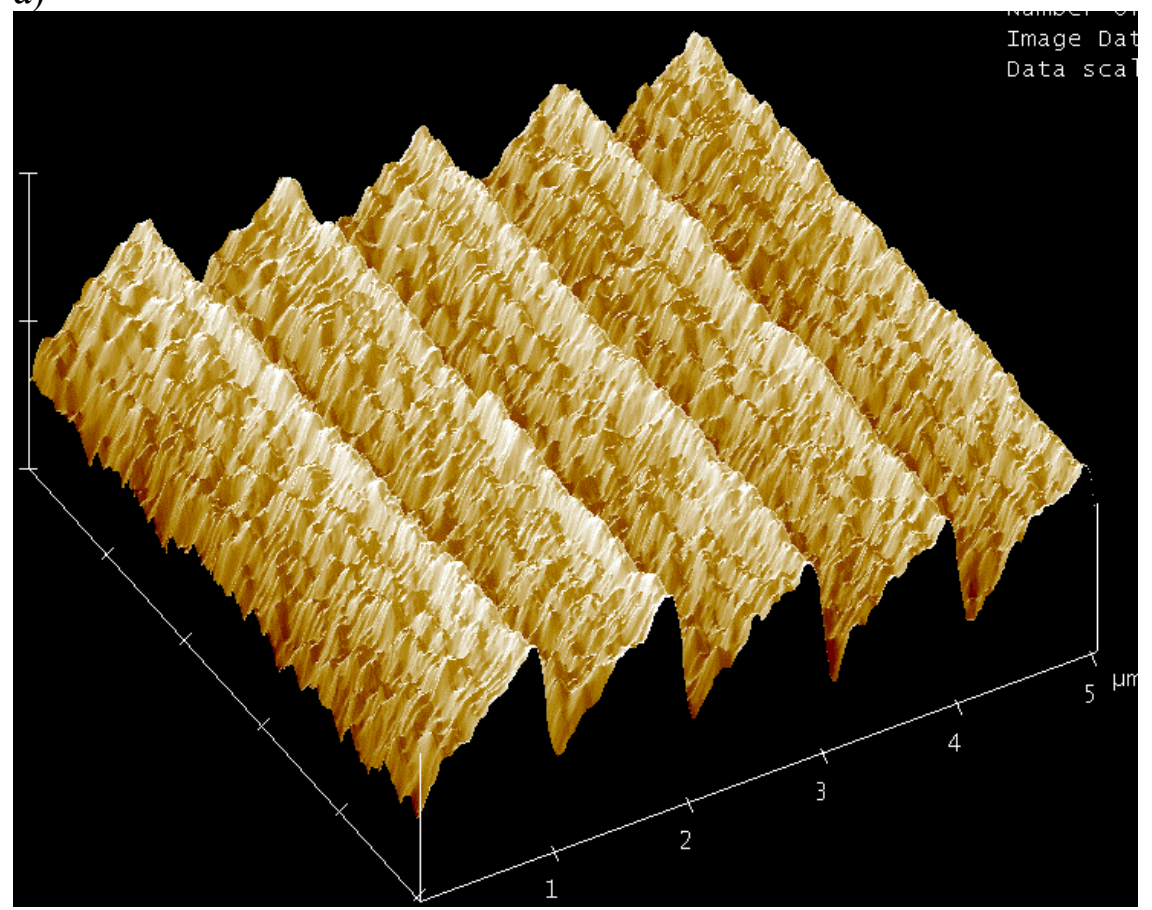

b)

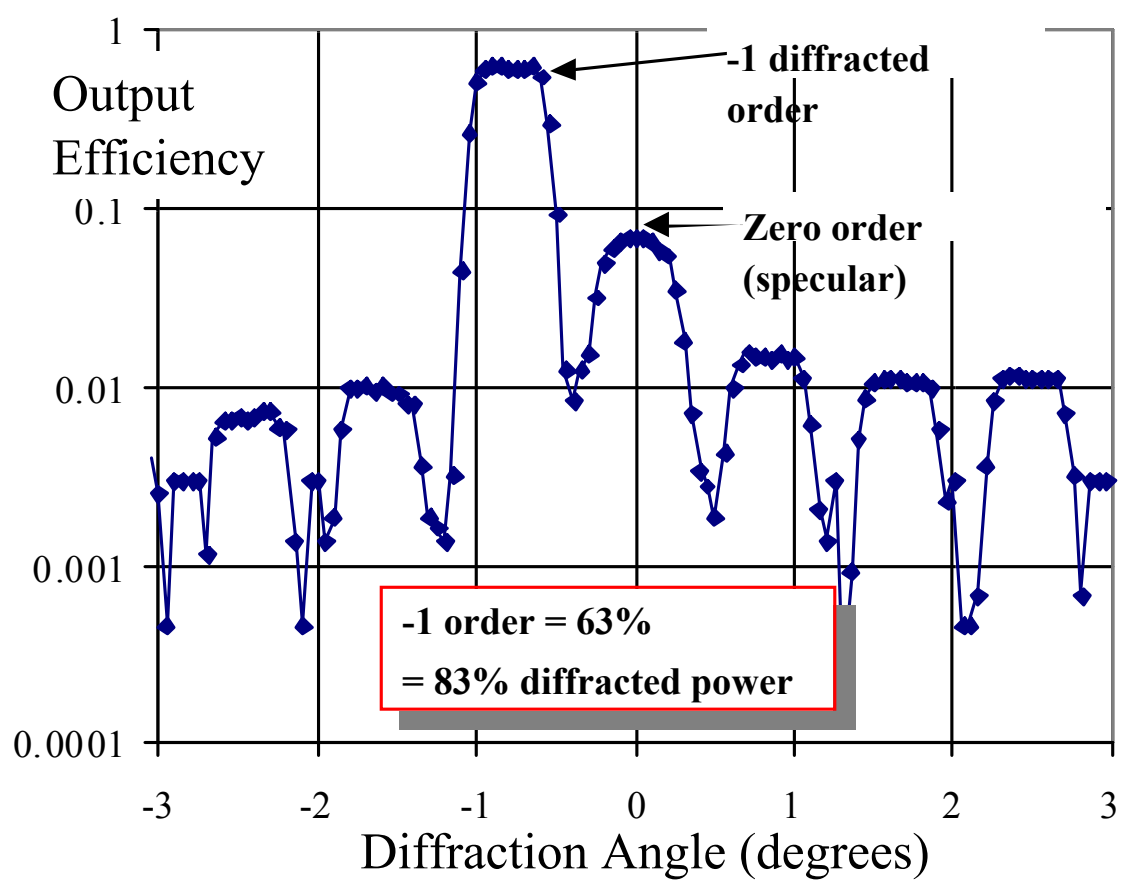

Figure 7. a) AFM image of a multilayer coated grating with RMS roughness of $0.5 \mathrm{~nm}$. b) Diffraction data obtained from the grating in a). 